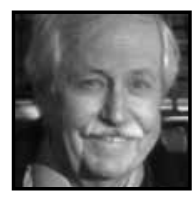

\title{
Getting Intelligence Into the Minds of People
}

\author{
Robert W. Lawler
}

\begin{abstract}
In conversation, Seymour Papert once asked me, "What's the point of studying Artificial Intelligence if not to get intelligence into the minds of people?" His question inspires my juxtaposition of explorations of Natural Learning and Constructed Personal Knowledge. Since "you can't learn about learning without learning about learning something," ${ }^{\prime 1}$ the analyses will proceed with two examples. The first, focused on strategy learning at tic-tac-toe, concludes that learning depends on specific relationships among the elements of the context in interaction with processes of incremental cognitive change. The second analysis, focused on mastering a solution for Rubik's Cube, argues the importance of reformulation of representations as a strategy for learning in more complex situations, and that the integration of multiple modalities of representation can be a key to "getting the intelligence into the minds of people."
\end{abstract}

\section{Introduction}

n Sciences of the Artificial, Herbert Simon (1969) introduced his discussion of "The Psychology of Thinking" with a novel perspective on agent-context interaction:

We watch an ant make his laborious way across a wind- and wavemolded beach. He moves ahead, angles to the right to ease his climb up a steep dunelet, detours around a pebble.... Thus he makes his weaving, halting way back to his home.... Viewed as a geometric figure, the ant's path is irregular, complex, hard to describe. But its complexity is really a complexity in the surface of the beach, not a complexity in the ant.... An ant, viewed as a behaving system, is quite simple. The apparent complexity of its behavior 
over time is largely a reflection of the complexity of the environment in which it finds itself.... (p. 63)

Simon concludes that his aim was exploring a similar vision of behavior, but with "human being" substituted for "ant." Over many years he pursued that aim, especially with his collaborator Alan Newell. In a broad and detailed case study of my first daughter's learning, I captured a complete history of her play at tic-tac-toe. Although simpler than chess and various puzzles that Newell and Simon (1972) explored, ${ }^{2}$ tic-tac-toe touched upon some of the same issues that were grand themes in the early days of Artificial Intelligence research, such as representing knowledge, strategic play, and interactions of thinking and environment. With further inspiration from psychological observations and the epistemological focus of Piaget, and from the data-orientated ecological psychologists, Barker and Wright (1971), I was able to exploit my case study material for the computational generation of paths of learning. Together, these enable a new idea of what makes discovery-learning possible and memorable (computer modeling turns this verbal "idea" into a worked example, with process detail).

\section{Natural Learning}

Many people believed, years ago, that computer modeling of student knowledge would enable the development of computer tutors sufficiently intelligent that they could replace poor or non-existent teachers. ${ }^{3}$ That engineering effort has largely succeeded and been absorbed into the technologies of our day. The scientific frontier of Artificial Intelligence, in the Minsky paradigm, ${ }^{4}$ focused on how knowledge could function (intelligence) and change (learn) through failures and repairs in articulated (specified and organized) knowledge structures.

What was most wonderful in my human case study 5 is seeing how learning can occur through serendipitous successes-events at least as important as failures-because such learning exemplifies learning through side effects, which may be more common for people than learning through the analysis of process failures, as in debugging. If recall of surprising successes can be reformulated as a new plan for future action, the process can be a prototype for Natural Learning, processes by which we all learn a bit more than we knew before about the business of everyday life; in Artificial Intelligence (Al), such knowledge is called "common sense." Exhibiting such processes in extensive detail was the agenda of the project in which SLIM 
(Strategy Learner: Interactive Model) was developed. ${ }^{6}$ Our effort was to use information from the child learning case study for guiding our knowledge representation, for following the development of strategies, and for examining the interplay of such development and the possibilities for learning based on specific experiences of play. The final focus was on the question of what it was that made learning possible at all, given limited initial knowledge and weak learning mechanisms.

\section{Learning Landmarks in the Human Case: (1)}

At the start of the study, Miriam played solely in a tactical way. Her moves were focused on selection of cells by preference for the center cell if available, then any corner, with side cells moves as a last resort. Although she knew forming forks was desirable (we called that "getting two ways to win"), she did not show any notion of how to achieve a fork. This changed after one specific incident:

I visited the Boston Children's Museum with Miriam, Robby, and his friend John. One single incident made a tremendous impression on Miriam and markedly influenced all her subsequent play. John and Miriam both played tic-tac-toe against the Museum's computer. When they fell in the pitfall of a side response to the computer's invariable middle opening, they were regularly defeated. The computer - which could be accurately described as playing with the Newell and Simon rules, always responded in the corner to their middle openings, so the children's best game was no better than a draw. Eventually Robby got a turn (the others peering over his shoulder) and opened with a corner move. The game unfolded... and Robby won. John and Miriam were astounded. Robby clearly owned some powerful knowledge. Since he was glad to share that knowledge with them, that one three-corner fork entered Miriam's repertoire and exerted a dominant influence in the development of her play. Tic-tac-toe became a game of frequent choice as The Intimate Study began.... This particular form of the game is my archetypical example of strategic play, quintessentially a game length plan for the single player -- as contrasted with the single cell move preferences, either choices or responses, of tactical play... ${ }^{7}$

Miriam now had a pattern-oriented goal but no plan to achieve that pattern. Play with Rob degenerated to negotiation (taking turns at winning), complaining (when blocked), and cheating (to circumvent blocks). I stopped their play against each other and became Miriam's primary opponent. Her play was "egocentric," in the sense of being focused on her goal, not because she was insensitive to her opponent's 
possible moves, but because she did not comprehend and had no way to deal with the opponent.

\section{Learning Landmarks in the Human Case: (2)}

The second major advance was the beginning of mental play. This grew out of a practice we called "Turning the tables," thus: When we played and Miriam lost a game, I urged her to replay the same game with our roles reversed, i.e., we would replicate a game, move for move, which she would win in the replay because she was playing as the opponent to whom she had lost. Mental play began this way

At one point, I was called away from play by our doorbell and asked the two children not to play any games until I returned.... Coming back, I found Miriam had been playing tic-tac-toe against herself, in her words, "making smart moves for me and the other guy."

Her multi-role play worked by setting herself the strategic goal and assigning tactical play to "the other guy." This split was eventually the foundation for the next landmark.

\section{Learning Landmarks in the Human Case: (3)}

At the end of the study, Miriam invented a strategy different from a variation of her archetypical three-corners fork.

Reviewing her play of the intervening years, Miriam stated that she played rarely and mostly against herself (30 games in two years was her estimate). As I introduced my objective, Miriam proposed one of her own: to apply a newly discovered strategy against me. When I asked her to discuss it with me, she responded, "No way! Then whenever I play you'll know my tactics."

Miriam's new strategy, the Middle and Corners Fork, is nonetheless clear from her play on the game and ... in a final comment on the discovery of this new strategy, Miriam added that she had stopped the game and only days later noticed the fork created with her third move. (This is verified by the appearance of the original game: the first six markers were made in brown crayon and the last winning move in ball pen ink.) 


\section{Conclusions for Modeling}

In relating case study details to computing models, one might use those details much as one uses boundary conditions to specify the particular form of a general solution to a differential equation. So we use the psychological study here, as a foundation for the representations used in the models, and as justification for focusing on key issues: the centrality of egocentricity in cognitive self-construction and the particularity of the naive agent's knowledge.

What I drew for representation from these naturalistic observations was:

1. a decision to represent separately a goal as a pattern, and a plan as a list

2. a willingness to see new learning as recognition of a new strategy, after the fact, by reflection on the player's own actions.

3. a recognition that mental play is necessary for analysis because simulation of "the other guy" implies the player can understand the opponent's reasons for action, as compared to some "mysterious and incomprehensible other" with whom one can cope only by social interactions or wishful thinking.

With respect to focusing on the development of a strategy, I review here the one simplest strategy to invent, to exhibit the interaction of knowledge with opportunities within the intellectual "landscape." With respect to better grasping that "landscape" of learning in this mental environment, we used a computer-embodied model to generate the suite of all games that could be played from a single opening move, then to examine those games in which the opening player experienced an unplanned win. By cataloging the sets of games which could be won without a plan, based upon a given known plan in the model's repertoire, we construct genetic pathways of plan descent.

\section{Using Simulations to Track Paths of Learning}

\section{Learning Through Interaction}

One virtue of machine learning studies is that they allow us no "miracles." They can completely and unambiguously cover some examples of learning with mechanisms simple enough to be comprehensible. Further, the computer's aid in systematically generating sets of all possible conditions helps liberate our view of what possible experiences might serve as paths of learning. When we do generate all possible interactions through which learning might occur, we can more 
thoroughly explore alternate paths and the suite of relationships among elements of the ensemble.

Strategies for achieving specific forks are the knowledge structures of SLIM (Strategy Learner, Interactive Model). Each has three parts: a Goal, a sequence of Actions, and a set of Constraints on those actions (each triple is thereby a GAC). We simulated operation of such structures in a program where SLIM plays tic-tactoe against variations of REO (a programmed Reasonably Expert Opponent). REO is "expert" in the sense of applying uniformly a set of cell preference rules for tactical play. ${ }^{8}$ ) Applying these strategies leads to moves that often result in winning or losing; that leads to the creation of new structures, by specific modifications of the current GACs. The modifications are controlled by a small set of rules, so that GACs are interrelated by the ways modifications can map from one to another. ${ }^{9}$

To evaluate specific learning mechanisms in particular cases, one must examine and specify which forks are learned from which predecessors in which sequence and under which conditions of opponent cell preferences. The simulation avoided abstraction, in order to explore learning based on the modification of fully explicit strategies learned through particular experiences. ${ }^{10}$ The results are first, a catalog of specific experiences through which learning occurs within this system and second, a description of networks of descent of specific strategies from one another. The catalog permits a specification of two desired results: first, which new forks may be learned when some predecessor is known; and second, which specific interaction gives rise to each fork learned. The results obviously also depend on the specific learning algorithm used by SLIM.

Consider how SLIM can learn the symmetrical variation to one particular fork (refer to the cell, plan, and game representations in Figure 1, below). Suppose that SLIM begins with the objective of developing a fork represented by the Goal pattern in the set $\left\{\begin{array}{ll}1 & 3\end{array} 9\right.$ \} and will proceed with letter moves in the sequenced plan [1 93 ] (as in Figure 1). SLIM moves first to cell 1. REO (numbers) prefers the center cell (5), and moves there. SLIM moves in cell 9, according to plan. When REO's second move is to cell 3, SLIM's plan is blocked. The strategic goal $\left\{\begin{array}{ll}3 & 9\end{array}\right\}$ is given over-but the game is not ended. SLIM, now playing tactically with the same set of rules as REO, moves into cell 7, the corner cell remaining. Unknowingly, SLIM has created a fork symmetrical to its fork-goal. SLIM cannot recognize the fork. It has not the knowledge or information to do so. What happens? REO blocks one of SLIM's two ways to win, choosing cell 4. SLIM, playing tactically, recognizes it can win and moves in cell 8 . This is the key juncture. 


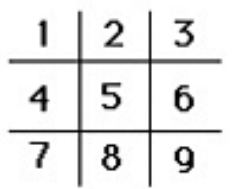

numbered cells

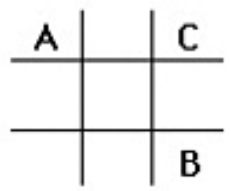

egocentric plan

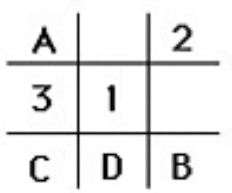

accidental win

Fig. 1: Cell, plan, and game representations

SLIM is programmed to recognize "winning without expecting to do so" as a special circumstance. Even more, SLIM assumes that it won through creating an unrecognized fork (otherwise REO would have blocked the win). SLIM takes the set of its first three moves as a pattern for a fork. That pattern $\left\{\begin{array}{ll}1 & 9\end{array}\right.$ \} is made the goal of a new GAC. SLIM examines its known plans for creating a fork (there is one, [1 93]) with the list of its own moves, executed in sequence before the winning move was made [ $\left.\begin{array}{ll}1 & 9\end{array}\right]$. The terminal step of the plan is the only difference between the two. SLIM modifies the prototype plan terminal step to create a new plan, [1 9 7]. SLIM now has two GACs, two forking strategies, for future play. ${ }^{11}$

The complete set of results involves consideration of all paths of possible learning, even those deemed unlikely a priori, and concludes with the complete specification of all possible paths of learning every fork given any fork prototype. For corner opening play, the first six GACs form a central collection of strategies. Their interrelations can be represented as trees of derivation or descent (as shown in Figure 2).

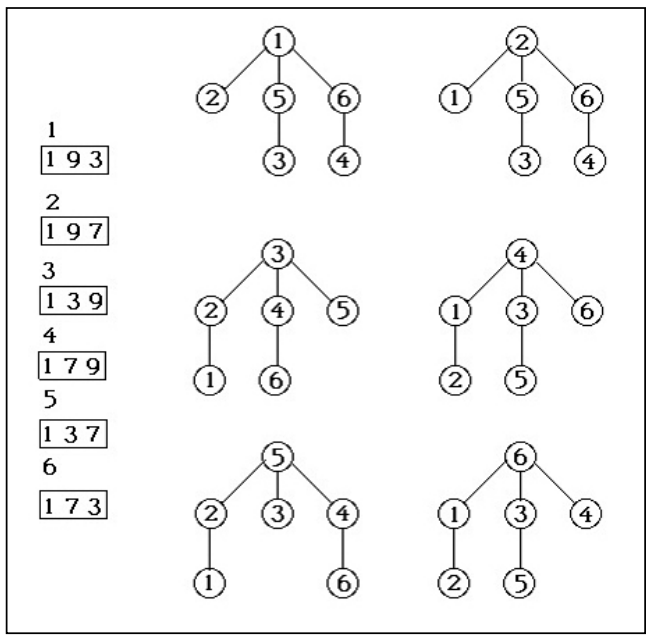

Fig. 2: Plans learnable from the plan specified as the top node of the descent trees 
The tree with strategy three as top node (beginning with only GAC 3 known) may be taken as typical. Play in five specific games generates the other five central GACs, i.e., five central strategies. The specialness of the six central nodes is a consequence of their co-generatability. Some of those are directly generatable, can generate each other (such as GACs 1 and 2); they are reciprocally generatable. Some lead to each other through intermediaries (such as GAC 1 and 3); they are cyclically generatable. For these six central strategies, the trees of structure descent can fold together into a connected network of descent whose relations of co-generativity are shown in Figure 3.

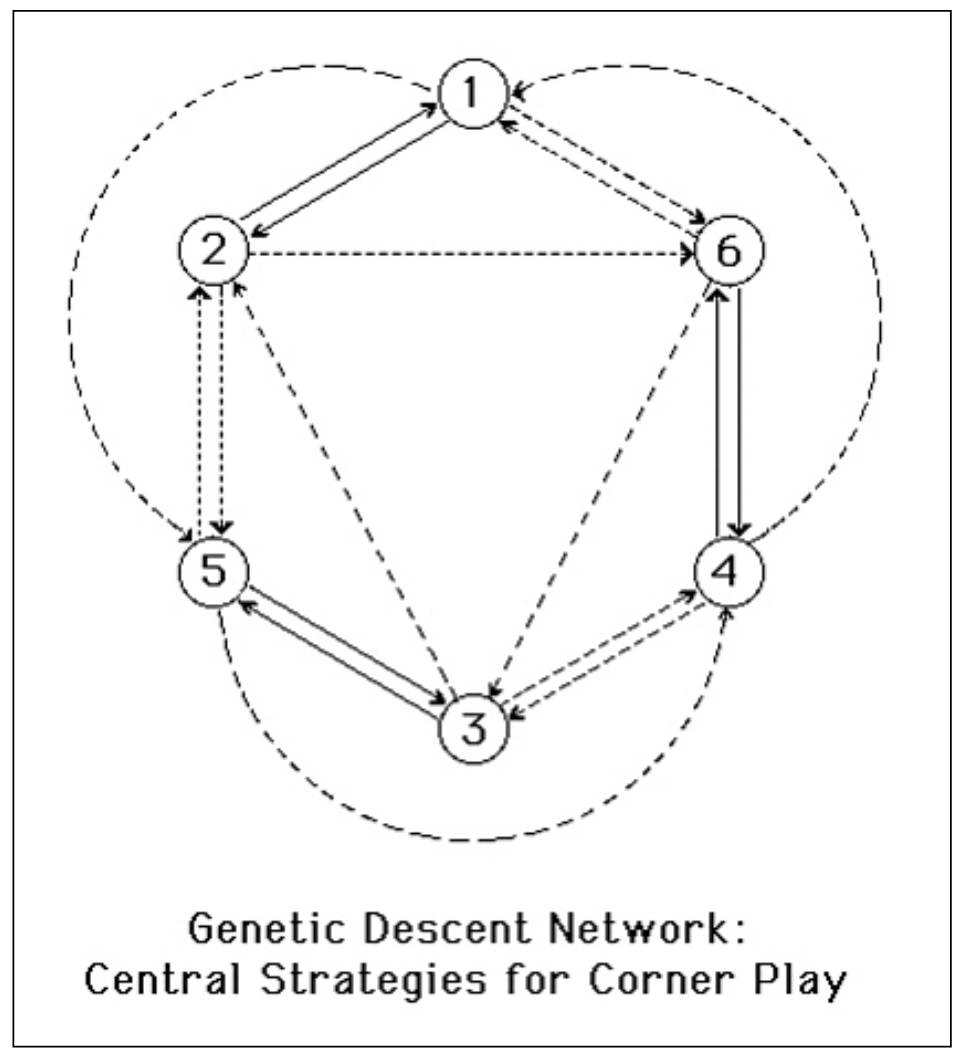

Fig. 3: Genetic descent network: Central strategies for corner play

The form of these descent networks is related to symmetry among forking patterns. But they include more: they reflect the play of the opponent, the order in which the forks are learned, and the specific learning mechanisms permitted in the simulations. These descent networks are summaries of results. 


\section{Reflective Summary}

Working with SLIM started with the general principle that learning happens through interaction. The model was constructed to represent the behavior of both the learner and the opponent in explicit detail with specification of representations and learning algorithms giving the notion a precise meaning. Through focusing on particular detail, we are exploring how knowledge can be extended by specific changes without abstract generalization. The question is of general interest if one admits that particularity and egocentricity are common characteristics of novice thought.

In analyzing the possibilities for learning, the basic principle applied is to try all cases and construct an interpretation of them. There are many paths of possible learning, some central and some peripheral. In SLIM, the criterion of centrality is co-generativity. We first aggregate results of all possibilities in a fully explicit manner. SLIM highlights co-generativity, represented by those connections in the central group (strategies 1-6) permitting each one to be learned no matter which is adopted as the prototype fork. Peripheral strategies ${ }^{12}$ are rarely learned because they can be learned in few ways. The aggregation of trees is systematic and constructive though not formal: one pulls together the empirical results of exhaustive exploration (trees of descent in Figure 2) into a new representation scheme (the genetic networks of descent in Figure 3).

Through the aggregation and reformulation of results, a new generality was achieved which suggested an idea, call it a "learnability principle," that co-generatability of related but variant knowledge forms is what makes learning possible in any domain. This principle would support knowledge stability in minds with reconstructive memories, such as Bartlett (1932) suggests humans have. This idea-identifying the learnability of a domain with its connectedness-is a direct consequence of the knowledge state transformations being the learning algorithms of the system. One may paraphrase the situation thus: "you'll learn where Rome is, if all roads lead there."

In tic-tac-toe, we have focused on a two dimensional puzzle, size $3 \times 3$. At first

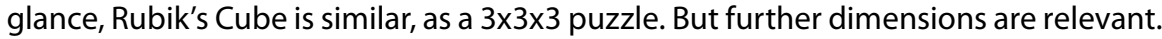
Color enters consideration as well as constraints implicit in the interior mechanism. Let's look at the situation generally before plunging into puzzle solution. 


\section{Constructed Personal Knowledge: Mastering a Solution to Rubik's Cube}

Over 350 million Rubik's Cubes have been sold worldwide. When a cube is "solved," each of the six faces has a single color. There is one such state. There are many more unsolved states. ${ }^{13}$ There are different methods for solving this puzzle; typically one solution is provided as a guide when a puzzle is purchased, e.g., a "7 Step Solution Guide." Many people take up this puzzle to prove they are "smart."14 Often these cubes remain disordered and are set aside, and the confident problem-solvers are defeated. Rubik's Cube remains an opportunity for a personal learning experience and more, an example of activities involved in learning to solve, to understand, and to remember a puzzle solution that many find too difficult.

\section{The Challenge Rubik's Cube Presents}

Rubik's 3x3x3 cube first appears to be an assembly of 27 different little cubes (call them "cubies") linked together internally. The mechanism takes up the central cubie and the six face-center cubies. These seven never move (except by cube rotation). Thus there are 20 moveable pieces, i.e., 12 edge cubies and 8 corner cubies.

\section{What makes the puzzle engaging}

As purchased, the cube comes in its solved state. This proves it can be solved. The cube can be held in your hand; it is even sold with a solution booklet. Everything about it is familiar, except the mechanism inside. Clever people conclude, "I can do this."

\section{What makes the puzzle hard}

Multiple modalities: recognizing visual patterns is essential to solving the cube; planning requires foreseeing how sequences of moves change multi-facial patterns.

Sameness of views: it is easy to get lost in regards to orientation.

Sameness of operations: it is easy to lose track of "where you are" in move sequences. Interactions: changing the state of any face, edge, or cubie ALWAYS impacts others. Progress requires re-balancing focus from "placing" cubies to protecting those in place.

Plans are hard to remember: mid-solution sequences typically involve 6-8 steps; backing out is hard; if confused, you need return to a state where you can restart.

Apparent disorder can mask closeness of the solution. 


\section{Becoming Competent at Solving Rubik's Cube}

Most of us are not going to invent our own solution. In this analysis, we will adopt the solution provided at purchase in the booklet, "7 Step Solution Guide." Our goal is an exercise in understanding that solution with sufficient recall of the details so one can apply it flexibly without any external support.

The "Solution" booklet specifies one common way people refer to the faces of the cube [front, back; right, left, upper, down] and names the operations on the faces. For example: " $F$ " means rotate the front face one quarter turn in a clockwise direction; "Ui" means rotate the upper face one quarter turn in a counter clockwise direction. An "algorithm" can be expressed as a list of operations, thus: [U R Ui Ri Ui Fi U F]. There is no way I can remember such a list of actions and execute each operation without making errors. I need to develop some other way of thinking that I can understand, remember, and execute without errors. These notes on my ways of thinking may suggest how you can create a way of thinking you can remember.

\section{A Common Strategy for Approaching Such a Problem}

Determine what does not change, what changes, how changes can be undone, and how the problem can be broken into parts. Since the 6 face-center cubies never change places, they serve as reference points. The 12 edge-center cubies can appear in any of 12 locations, with 2 orientations; there are 8 places for corner-cubies and 3 orientations.

A single twist of any face can be undone by the inverse twist, e.g., Fi negates F. For a sequence, the order must be reversed while replacing each operation with its inverse, e.g., [Ri Ui] negates [U R]. Negation quickly gets tricky for longer sequences of twists. Additionally, after any single twist of a face, the prior state of the cube can be restored by completing a cycle of restoration, e.g., the state prior to move [U], can be restored by repeating the twist $[U]$ three times more [U U U]. With sequences of twists, restoration by repetition becomes impractical very quickly.

Can the problem be broken into manageable sub-problems? That depends on what you can manage. It asks, "What can you recognize and remember?" The "7 Step Guide" is confusing. Manageable, for me, demands some measure of progress, "achieved goals" that can be remembered. And it is very helpful to be able to characterize "manageable" sub-problems. I see the Rubik's cube solution in 3 initiatives:

Solve the bottom layer: this is the easy problem. (Steps 1 and 2)

Solve the middle layer: this is the tricky problem. (Step 3)

Solve the top layer: this is the challenging problem. (Steps 4-7) 


\section{Part I: Solving the Bottom Layer}

The booklet's first goal: solve the green cross. ${ }^{15}$ This is easy since you don't have to think about cubies that do not have a green side. Turn the cube so the green center cubie is on the upper face. Find any edge-cubie with a green face. Rotate that face so the green face of the edge-cubie is next to the green center-cubie. Rotate the top (green-centered) face so that the edge-cubie's second face is next to the centercubie of the same color. Good start, you have the first edge-cubie in its place!

Next, put a second green edge-cubie in the "same" relation to the green center-cubie and its other face color center-cubie-without permanently moving the first edge-cubie away from its matching side face. Get all four green-edge cubies in place. You can do it by trial and error, for the second, third, and fourth "greenand- $X$ " edge cubies. (This will take a while, but it's easy and it's good familiarization and practice.)

Fixing orientation problems: it often happens that some of these cubies are oriented so the green color of the cubie is on the "wrong face" (the one adjacent to its second color center-cubie). The booklet presents a 4-move procedure to flip this cubie to the goal orientation; [Ri U Fi Ui], where the lower case "i" means: "for this move, rotate the specific face counter-clockwise." To explicitly specify the rotation-sense of moves, we represent a clockwise twist with a " + " sign, and counterclockwise twists with a "-". Thus: [Ri U Fi Ui] $\rightarrow$ [R- U+ F- U-]. "How to view the cube" is important too.

See: 3 faces visible, green on top; edge-cubie flip-target on right face.

Do: procedure: [R- U+ F- U-]; after, verify the target cubie's orientation.

Review: why does this work? You have to be able to answer for yourself. I find it helpful to reverse the procedure by $[\mathrm{U}+\mathrm{F}+\mathrm{U}-\mathrm{R}+]$, thus restoring the prior state, then to step through the procedure again, carefully watching the target cubie to see how each move affects its orientation and relation to the other cubies.

With the first five cubies in place, if we can do the four corners, we'll have a direct solution for the bottom layer! How to do it? One cubie at a time.

Reflection: notice that we have modified the representation of the operations performed on the faces of the cube. This is done to explicitly specify an aspect (sense of turning) of the operation. This is important because it brings into concrete 
specification an element of the operation that otherwise would be "assumed as a default," i.e., remembered.

The greencross, step one's now "achieved goal," marks a significant change. One must begin to think about changes using procedures that do not relocate other cubies already in their target positions. With the green face on top, the booklet proposes locating a cubie in the current bottom then rotating that bottom layer until that cubie is directly under the place it must go; call that the cubie's home position. The goal is to get the four green corner-cubies home. The booklet proposes [R-D-R+ $D+]$. This rotates the corner-cubie's home position into the face opposite the green face and twists that opposite layer to insert the target cubie into the "home" position, then restores the home position to the green face. The booklet directs execution of this procedure 1,3 , or 5 times. Why is this number variable? It's neither obvious to a novice nor explained.

The number of execution required depends on the original orientation of the target cubie when under the home position. There are three possibilities:

See: 3 faces visible, green on top; the target cubie under its home.

$>\quad$ if its green color is on the front face, then do variation 1 [D- R- D+ R+]

$>\quad$ if its green color is on the right face, then do variation 2 [D+F+D-F-]

$>\quad$ if target-cubie's green color is the bottom face, Do setup procedure [R- D$\mathrm{D}-\mathrm{R}+\mathrm{D}+\mathrm{]}$; it puts the green color on the right face, under its home. Then do procedure variation $2[\mathrm{D}+\mathrm{F}+\mathrm{D}-\mathrm{F}-]$.

Review: why does this work? The symmetrical variations are "the same" in the general sense of bringing the home position to the bottom layer where a different cubie can be inserted into the target edge. When the target-cubie's green color is on the bottom, the setup procedure changes its position to the right face where variation 2 is used.

There is an issue here which some would see as one of principle, i.e., is it better to represent knowledge by a uniform procedure which is harder to understand or is it better to represent knowledge by multiple alternatives which may be easier to learn and remember? I see the issue as one of choice, yours, which depends on your personal style and established ways of thinking. Do you understand why these procedures work? ... Yes? Congratulations then, you have mastered the first layer of Rubik's Cube! 


\section{Part II: Solving the Middle Layer}

When you turn the cube, setting it down on the green face, you will see that the middle layer is already "half-solved," because all the face-center cubies are where they belong (they always are). What's tricky in the middle layer is inserting four edgecubies in their own home positions, with the proper orientation. Of course, this must be done without changing any cubies in the bottom layer, except temporarily.

The typical situation is that an edge-cubie belonging in the middle layer can be placed over a color matching face-center cubie. Assuming the cube is oriented so that the home for the target cubie is on the vertical forward edge, it must be true that the "unmatched" face of the edge cubie is either on the current right face or the current front face. These symmetrical states require solution by symmetrical procedures. Look for any edge-cubies that are already in their home positions. If there is one, turn the cube so the next vertical edge is forward. Observe the colors of the front and right center-cubies. Find an edge-cubie that has both those colors on its faces. Choose that cubie and rotate the top layer so that the side color of the chosen cubie matches either the front or right face-center cubie color. The booklet specifies an eight-step procedure and a symmetrical variation to insert that chosen cubie in its home.

For me, eight steps is too many to remember. The risk is significant confusion and errors, which require starting over. I need an idea that appears simpler than either [U R Ui Ri Ui Fi U F] or [Ui Fi U F U R Ui Ri]. But think about it. If the states are symmetrical, then there must be some symmetry in the procedures which reveal their internal structure.... Notice that the last four moves of the first procedure are identical to the first four moves of the second, and the last four moves of the second are identical to the first four of the first procedure. I can represent this as two "sub-procedures:"

$>$ for a target cubie on the right face, do: sub 1 [U- F- U+F+] sub 2 [U+R+U- R-]

$>$ for a target cubie on the front face, do: sub $2[\mathrm{U}+\mathrm{R}+\mathrm{U}-\mathrm{R}-]$ sub 1 [U- F- U+ $\mathrm{F}+]$

Reflection: this specification of sub-procedures is based on a need to understand, resolved by observations on the structure manifest in a set of operations. This defines a "manageable" sub-problem by aspects of the problem, as distinct from the needs and limitations of the problem solver. 
Remembering these sequences of four moves is still hard. We cannot change the specific moves or their sequence, but we can group the moves by what sequences have in common, their "sense of twist" (signs plus or minus, meaning clockwise or counter clockwise), thus:

$>\quad$ right face cubie: sub $1[\mathrm{U}-\mathrm{F}-\mathrm{U}+\mathrm{F}+]$ sub $2[\mathrm{U}+\mathrm{R}+\mathrm{U}-\mathrm{R}-]->[-\mathrm{UF}+\mathrm{UF}]$ [+UR -UR]

$>$ front face cubie: sub 2 [U+ R+ U- R-] sub 1 [U- F- U+ F+] $->$ [+UR -UR] $[-U F+U F]$

One can even pronounce the move sequences as syllables; this could aid recall, even though the syllables be non-sense. Now, wherever there is a middle layer home position not occupied by its color specified appropriate cubie, one specifies:

See: 3 faces visible, green on bottom; forward edge "empty"; chosen cubie above right or front face-center cubie.

Do: either the front face or right face procedure above, as appropriate.

Review: Think about what's needed to replace the forward edge-cubie while keeping the base layer cubie "under" it. In completing the bottom (green) layer corners, it was enough to "slice" a single cubie into an edge to complete a single color edge, then rotate that edge into the green layer. For the middle layer, one must assemble a two-chunk portion of the forward edge which can be rotated to the vertical in a single move. But that can only be done after the "two-chunk" forward edge is joined perpendicularly to the bottom layer edge which shares the corner cubie. Apply your visual imagination; understanding THIS point is worth all the time it takes you.

\section{Part III: Solving the Top Layer}

The "7 Step Solution" for part III involves four steps in this order:

1. solve the top (blue) cross

2. re-order the top edges

3. re-order the top corners (1)

4. re-orient the top corners (2)

The first three of these appear simple because they are designed to return to the initial state, except for movement of the targeted cubie. 


\section{Solving the Top (Blue) Cross}

This goal places top layer edge-cubies in the correct orientation (blue on the top layer). There are four such edge-center cubies. Either zero, two, or four of these will have the upper face (blue) color. If four, then the blue cross is already completed. The six moves of the procedure below flip two of these cubies with every execution, one in each three-step sub-procedure:

See: 3 faces visible, green on bottom; turn the cube until you see the top center cubie blue, or a blue arrow (3 cubies) pointing at you, or a blue bar ( 3 cubies) from the left to the right face.

Do: procedure: sub 1 [+FRU] sub 2 [-RUF] once, or two times if needed, until you see a blue cross (possibly with more blue cubies) on the top face. THEN turn the top layer so that a maximum number of top-edge cubies are adjacent to the same-colored side face-center cubies.

Review: If the top layer shows none or 2 edge-cubies blue on top, this implies there are four or two top-edge cubies that are blue on the side. The procedure flips the toplayer edge cubies then restores the lower level layers.

\section{Re-Ordering the Top-Edge Cubies}

Since now all the top-edge cubies will be of the same face color, we can distinguish them by naming each for its side face color. You can always match at least one top-edge cubie with its same-color side face-center cubie. Choose one and consider this the head of a list, e.g., yellow. As I rotate my cube clockwise on its green base (viewed from the top), the sequence of side-face colors is [yellow orange white red]. To solve the top edges, it is necessary to put every top-edge cubie in alignment with its side-edge color-matching center-cubie. If I list in sequence the topedge cubie face colors during the same cube rotation, I might see they are [yellow orange red white]. The order of the last two top-edge cubies needs to be swapped. The booklet provides an eight-step list of moves to do it [R U Ri U R U U Ri]. Using exactly the same moves, I describe it this way:

See: 3 faces visible, with green on bottom, orient the cube so that the two cubies to be swapped are to the left of the forward vertical edge.

Do: procedure: sub 1 [+RU-R+U] sub 2 [+RUU -R]. If a second set of top edge-cubies needs to be swapped, do it again. No more executions should be needed. 
Review: The procedure matches each side face with its top-edge side-color cubie. One is always possible. Two swaps are all that is needed to re-order the other three.

What remains to complete part three? Get all top-corner cubies in appropriate corners (1), and make sure each top corner cubie is oriented to color match its side faces (2).

\section{Re-Ordering the Top-Corner Cubies}

As with the top edge-center cubies, where we needed a procedure to reorder the sequence to get them "in the right place," so here we also need a procedure to sort the corner cubies into their appropriate home positions. This is how I represent it:

See: 3 faces visible, with green on bottom; if any top-corner cubie is in its appropriate home position, rotate the cube to make it top of the forward edge.

Do: procedure: [+UR -UL][+U -RU +L] (this re-sequences the corners list; you may need to do it twice).

$>\quad$ if there is no corner cubie "in the right place," execute this procedure once as a setup procedure, then follow "what to do." (This implies a maximum of three executions).

\section{Flipping the Top-Corner Cubies}

The solution's nearly complete. Go on carefully. It would be a shame to mess it up now!

See: three faces visible, with green on bottom, red as front face. Do NOT change the orientation of the cube until the solution is complete.

Do: rotate the top layer until a cubie needing to be flipped is top of the vertical edge;

$>$ do procedure: [-RD +RD] 2 or 4 times, pausing when the cubie has been flipped.

$>\quad$ Rotate the top layer to bring another cubie needing to be flipped to the top vertical edge. For each cubie needing to be flipped, do procedure [-RD+RD] 2 or 4 times, pausing when the cubie has been flipped. (Expect the lower layers of the cube to appear "messed up" at this point. Moves to flip the last corner cubie, done faultlessly, will restore order to the lower layers as well as completing the top layer solution.) 
When no more cubies need to be flipped, align the top edge pieces with their side color matches. The solution should be complete; stop.

Review: During this final stage, the configuration of cubies can still appear to be a mess. One might think a miracle would be needed to solve the cube at this point... and then a miracle happens: at some point while performing this procedure, the cube "solves itself." Any one who has succeeded in solving the cube will know from experience that this DOES happen. A novice will have to take the statement on trust. Best for everyone is an understanding of why the inter-dependencies of cubies, moves, and patterns make this conclusion necessary and obvious.

\section{Why Does the Double [-RD +RD] Work?}

Let's assume the cube is now solved. How did that actually happen? Talk of "miracles" is a metaphor here. Such "happy accidents" are excuses we accept as explanations if we cannot or will not pursue deeper understanding. HERE is an opportunity to understand complex inter-dependencies, if you want to.

A key point is one never need to do so in full detail, so long as you are satisfied that: when you change one cubie, you do something similar to but different from that for other cubies in comparable positions, interconnection of the cubies guarantees eventual return of a solved state.

$>$ why "guarantees?"

$>\quad$ there are only FEW cubies in play (you can see the four corner cubies that are changed)

$<\quad$ the operation is designed to change the orientation of a single cubie, but all four in play are being changed at the same time.

The complete cycle of executions is six to restore the original location and orientation. As Groucho Marx once asked, "Who're you gonna believe? Me? or you own eyes?"

An execution of [-RD+RD] moves the target cubie to its front face diagonal corner. There are two ways to return that cubie to its home position. The first is to undo the execution by running the procedure in reverse, with the signs changed, that is, [-DR+DR]. This restores the cubie to its original location and orientation (but if it needs to be flipped, why do that?)

The second is to repeat the execution of $[-R D+R D]$ twice. This restores the location of the target cubie but changes the orientation, rotating the blue face from 
top to front. Then rotating it from front to right. Two more repetitions will move the blue face from right to top, then from top to front. Two final repetitions will restore the original orientation by moving the blue face from front to right then from right to top. These six repetitions comprise a cycle of restoration.

Think about what is happening with other cubies as you focus on the target. They are changed also as a "side-effect" of your actions on the target cubie. If you undo the procedure for the target cubie, you also undo the side effect changes to the other cubies.

But what happens when you repeat the procedures in a cycle of restoration? Since the six executions of $[-R D+R D]$ suffice to restore the location and orientation of the target cubie, the interdependence of the cubies' states, created by their mechanical connection, argues that pairs of executions will repeat location changes and triplets of pairs will restore the original orientations-when no errors are made. This explains "the miracle of the cube solving itself" in the final step.

\section{Succinct Solution Summary:}

1. solve the bottom layer: 1). by forming the green cross with edge-center cubies color-matched to corresponding face-centered cubies; 2 ). by bringing the corner-cubies home in the color matched orientations.

2. solve the middle layer: by making the bottom layer the "down" face then inserting four edge-center cubies in their doubly color-matched "homes."

3. solve the top layer: 1). by forming a blue cross with edge-center cubies colormatched to corresponding face-centered cubies (at need, swap edge-center cubies); 2). by bringing the corner-cubies home in the color matched orientations (at need, swap the corner-cubies); 3). flip faces of corner cubies for colormatching at need.

4. remember the procedures for each step of the solution.

\section{Remembering This Solution}

Mnemonics, enhancing recall with various schemes, has been a theme in our culture for a very long time. ${ }^{16}$ Today, the acronym "HOMES" helps school children recall the list of Great Lakes (Huron, Ontario, Michigan, Erie, Superior). The hopeful sentence "Good Boys Do Fine Always" may aid recalling the lines of the base clef. Cicero depended on tours through architectural sites to structure and recall his famous orations. My memory needs all the help it can get, so I developed a set of 
mnemonics to counter the confusion potential of Rubik's Cube (they're set out in Figure 4). They are useful to me because they connect to things I've long known, and are strange enough to minimize confusion. My main point is that they are personal, useful to me, of little use to anyone else, except as examples. Consider \#2, "Blue Cross is not frou-frou." Frou-frou means "frilly." This works for me because I was a computer consultant for Blue Cross nearly 50 years ago, and I know well their business attire was as staid as IBM, for whom I worked then.

Similarly, I enjoy employing Saint Anselm's Ontological Argument for the existence of God in mnemonic \#3, partly because his slogan "credo quia absurdum" (I believe BECAUSE it is absurd) has annoyed me for a lifetime. (A petty revenge, 'tis true.) The bizarre monologue ascribed to Tonto (\#4) - politically incorrect indeed, and reflecting the uncertainty of my children about my political leanings-reveals the key functional component. These mnemonics are formulae for translating a silly verbal construct into a procedure for twisting Rubik's Cube. This is clearest in \#1, which starts with algebraic sums of sub-procedure representation terms and ends with unconnected words whose syllable rhymes reflect the sequence of sub-procedure twists. Finally, mnemonic \#5, which is little more than a name for the results of the operation, is for me a connection to some kind of muscle memory for executing the procedure. This works for me too. You have to create mnemonics that work for you.

\section{Remembering This Solution:}

One needs to recall five physical-manipulation procedures for this solution, one with a symmetrical variant. Developing mnemonics related to personal experience or to something a bit silly can help recall.* These personal mnemonics are presented here, not as good examples, but to emphasize that YOU have to invent your own way of remembering these complexes of what you see, what you plan to $\underline{\mathrm{do}}$, and what you can remember. All three are critical to understanding and solving Rubik's cube. I wish you good luck!

1. for middle layer cubie insertion from:

the Right face: [-UF+UF] [+UR-UR] (encoding with rhyme)

the Eront face: [+UR-UR] [-UF+UF]

The algebraic sums of the sub-procedure symbols are zero. Moves for the "right" procedure rhyme with "duffer" and start with counter-clockwise rotation, while the "front" procedure rhymes with sheriff and starts clockwise.

2. for creating a "Blue Cross" in the top layer:

Form the BlueCross: [+FRU] [-RUF] (up to 3 times) Blue Cross is not frou-frou (It's more "FRU-RUF")

Once a consultant at Blue Cross, I know insurance office attire is not frilly; it's conservative business dress.

3. for reordering top-layer center-edge cubies:

Re-order Edges: [+RU-R+U] [+RUU-R]:

my mnemonics:

St.Anselm ["Are you, minus 'are', you ?"] i.e.["Are you you? minus 'are'?"]

This recalls an ancient argument about whether existence is a necessary attribute of a perfect God.

4. for reordering top-layer corner cubies:

Re-order Corners: [+UR-UL] [+U-RU+L\}

my mnemonics:

Tonte Changes Mind: [ "You right, not you left"] but then [ "You, not right you; more left"]

You can decode the direct correlation of moves and the bizarre monologue of this silly mnemonic.

As always, the trick is getting from the mnemonic back to the procedural move encodings.

5. for flipping top-layer corner cubies:

my mnemonics:

Flip Corners: [-RD+RD] $* 2$ or $* 4$, with cube position fixed Double (Comer Swap and Flip)

This mnemonic is merely a title, descriptive of the procedure result, not a link to other symbolic memories.

Fig. 4: Remembering the Rubik's cube solution 


\section{Conclusions}

Returning to Herbert Simon's metaphor of the ant on the beach, our aim to understand learning as a side effect of thinking in specific contexts is what requires us to "look inside" mental processes and their possibilities of change. The issue of representation is thus central for formulating and expressing Artificial Intelligence notions of thinking and learning. The study of Al should lead to considering the role representation plays in peoples' problem solving. What we learn from our worked example of mastering Rubik's Cube is that flexibility in applying representations may be key to being "smart enough" to solve Rubik's Cube. Deployment of various representations and functions, mastery of a variety of such "ways of thinking," is more important to intelligence than "native ability" (as sampled by traditional tests such as the Stanford-Binet). ${ }^{17}$

Archilochus, a soldier-poet of ancient Greece, wrote a famous couplet:

The Fox knows many tricks.

The Hedgehog has just one, but it's a good one. ${ }^{18}$

If we can glimpse in mnemonics some tricks of the Fox and see in the formal methods of mathematics the one good trick of the Hedgehog, note that Artificial Intelligence found its home between the Fox and the Hedgehog, applying strong methods where possible and weak methods when necessary. Thus I find it a very "human," opportunistic approach to solving problems. But are tic-tac-toe and Rubik's Cube serious problems, worthy of understanding and mastering, especially as they have involved us in such down and dirty, nitty-gritty problem solving? "YES!" is my resounding answer, since these examples approach "thought experiments" that permit us to ask:

What makes learning possible in a task domain? How can mathematical and epistemological ideas advance peoples' understanding?

And if it helps any reader take a neglected or embarrassing Rubik's Cube out of the closet, then note these exercises meet Feynman's criterion of worthwhile problems:

No problem is too small or too trivial if we can really do something about it.... The worthwhile problems are the ones you can really solve or help solve, the ones you can really contribute something to. A problem is grand 
in science if it lies before us unsolved and we see some way for us to make some headway into it. I would advise you to take even simpler, or as you say, humbler, problems until you find some you can really solve easily, no matter how trivial. You will get the pleasure of success, and of helping your fellow man, even if it is only to answer a question in the mind of a colleague..."19

If Feynman's view is adopted, we'll be "getting intelligence into the minds of people." ${ }^{20}$ Seymour would be pleased.

\section{Notes}

1. This is an echo, of course, of Papert's well-known quip that, "You can't think about thinking without thinking about thinking about something."

2. Human Problem Solving, Prentice-Hall, 1972. See especially section 4.

3. See the section "Intelligent Tutoring Systems," in Artificial Intelligence and Education, Vol. 1.

4. See Minsky $(1975,1988)$ and Sussman (1975).

5. The following summary is reported in greater detail in primary sources, "The Articulation of Complementary Roles," (Lawler, 1985) and "Consider the Particular Case" (Lawler, 1996), both online now at NLCSA.net as well as in print sources.

6. Undertaken with the guidance of Oliver Selfridge (1993) and some programming help by Bud Frawley at GTE's Fundamental Research Laboratory.

7. From "The Articulation of Complimentary Roles," at http://nlcsa.net/lc2b-tis-2/ Ic2b-analyses/lc2ba4/

8. REO's preferences are those common in tactical play: first, win if possible; next block at need; finally choose a free cell, preferring the center cell first, and then any corner cell and finally a side cell. See Human Problem Solving, Newell and Simon (1972). 
9. Subject to limitations based on rotational symmetry and the strategies adopted by the psychological subject, the generation of possible games was exhaustive. The analysis focused on won-games in which SLIM moved first in cell 1. See figure 1. The focus on corner opening play is based on the behavior of the subject and the relative variety of strategies such openings permit.

10. This focus of the models is precisely where the egocentricity of naive thought and cognitive self-construction of the psychological subject are embodied. The models are "egocentric" in the specific sense that no consideration of the opponent is taken unless and until the current plan is blocked. The psychological subject played this way. When a plan is blocked, SLIM drops from strategy driven play into tactical play based on preferences for cells valued by type (center, corner) and not by relation to others.

11. This form of learning by modifying the last term of a plan is one of two sorts; the other involves generating two possible plans based on deletion of the second term of a prototype plan. We know the forks achieved by plans [1 93 3] and [1 9 7] are symmetrical. SLIM has no knowledge of symmetry and no way of knowing that the forks are related other than through descent, i.e., the derivation of the second from the first. This issue is discussed in the longer versions of this text. See "On the Merits of the Particular Case."

12. These are detailed in "On the Merits of the Particular Case," in Case Study and Computing.

13. See Wikipedia general article on Rubik's Cube: http://en.wikipedia.org/wiki/ Rubik\%27s_Cube

14. See Wikipedia "Rubik's Cube in Popular Culture": http://en.wikipedia.org/wiki/ Rubik\%27s_Cube_in_popular_culture

15. I follow the booklet in choosing green to be the bottom layer, later as the "down" face. (The choice is arbitrary; it is easier to do the usual thing, if following a known solution.)

16. See Wikipedia articles on "Mnemonic" and "The Art of Memory": http:// en.wikipedia.org/wiki/Mnemonic, http://en.wikipedia.org/wiki/Art_of_memory

17. The second case study, LC2, at NLCSA, contains a public Binet Test Summary; it 
was summarized as an appendix in Computer Experience and Cognitive Development. An Analysis of Detail Data for the subject (at $6 ; 1 ; 17)$ exists under password protection at NLCSA.

18. Greek Lyrics, Richmond Lattimore, U. Chicago. 1960.

19. These comments, from a letter to a former student, show the wisdom of a man whose scientific brilliance is unquestioned. See "What Problems to Solve, by Richard Feynman" at http://genius.cat-v.org/richard-feynman/writtings/letters/ problems ["writtings" (sic) in the web address.]

20. One might also take seriously how rigorous was Feynman's notion of what it means to really SOLVE a problem; see his discussion of the experiments of "Mr. Young" in the legendary 1974 Caltech Commencement Address, "Cargo Cult Science" (1986).

\section{References}

Barker, R., \& Wright, H. (1971). Midwest and its children. Hamden, CT: Archon Press.

Bartlett, F. (1932). Remembering. Cambridge: Cambridge University Press.

Feynman, R. (1986). "Cargo Cult Science" in Surely You're Joking, Mr. Feynman! W.W. Norton. Retrieved from http://calteches. library.caltech.edu/3043/

Lattimore, R. (1960). Greek lyrics. Chicago: University of Chicago Press.

Lawler, R. W. (1985). "The Articulation of Complementary Roles," in Computer Experience and Cognitive Development, Ellis Horwood Ltd., Chichester, UK; John Wiley, New York. Retrieved from http://nlcsa. net/lc2b-tis-2/lc2b-analyses/lc2ba4/

Lawler, R. W. (1996). "Consider the Particular Case," in Journal of Mathematical Behavior, V.13, No. 2. Retrieved from http://nlcsa. net/lc0b-csc/lc0b-analyses/LCObA4/ (This analysis is a compacted form of the original discussion "On the Merits of the Particular Case," in Case Study and
Computing, Lawler \& Carley. Norwood, $\mathrm{NJ}$ : Ablex Publishing.)

Minsky, M. L. (1975). "A Framework for Representing Knowledge," in The Psychology of Computer Vision, P. Winston, (Ed.), McGraw-Hill.

Minsky, M. L. (1988). The society of mind. New York: Simon \& Schuster.

Newell, A., \& Simon, H. A. (1972). Human problem solving. Englewood Cliffs, NJ: Prentice-Hall.

Selfridge, O. G. (1993). "The Gardens of Learning: a Vision for Artificial Intelligence." Al MAgazine, vol. 14, no. 2., 36-48.

Simon, H. A. (1969/1996). The sciences of the artificial. $3^{\text {rd }}$ edition. Cambridge, MA: MIT Press.

Sussman, G. (1975). A computer model of skill acquisition. New York: Elsevier Science Inc. 


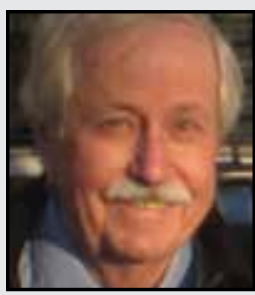

Robert Lawler studied science and engineering at Caltech and then literature and drama at Temple and Yale. He worked a decade with IBM as a software applications engineer before earning his doctorate for studies in Artificial Intelligence and Education with Marvin Minsky and Seymour Papert at MIT. He has worked in both in corporate and governmental research centers in Boston, New York, Washington, and Paris. During his Purdue faculty years, he consulted for Apple and OECD and held visiting positions at MIT, Radcliffe, Exeter (UK) and the University of Geneva $(\mathrm{CH})$. Since retiring as a Professor, he lives in Lake Geneva, Wisconsin.

\section{LINK TO:}

http://www.nlcsa.net 